\title{
Optical Properties of Dust
}

\author{
Aigen Li \\ Department of Physics and Astronomy, University of Missouri, \\ Columbia, MO 65211; LiA@missouri.edu
}

Summary. Except in a few cases cosmic dust can be studied in situ or in terrestrial laboratories, essentially all of our information concerning the nature of cosmic dust depends upon its interaction with electromagnetic radiation. This chapter presents the theoretical basis for describing the optical properties of dust - how it absorbs and scatters starlight and reradiates the absorbed energy at longer wavelengths.

\section{Introduction}

Dust is everywhere in the Universe: it is a ubiquitous feature of the cosmos, impinging directly or indirectly on most fields of modern astronomy. It occurs in a wide variety of astrophysical regions, ranging from the local environment of the Earth to distant galaxies and quasars: from meteorites originated in the asteroid belt, the most pristine solar system objects - comets, and stratospherically collected interplanetary dust particles (IDPs) of either cometary or asteroidal origin to external galaxies (both normal and active, nearby and distant) and circumnuclear tori around active galactic nuclei; from circumstellar envelopes around evolved stars (cool red giants, AGB stars) and Wolf-Rayet stars, planetary nebulae, nova and supernova ejecta, and supernova remnants to interstellar clouds and star-forming regions; from the terrestrial zodiacal cloud to protoplanetary disks around young stellar objects and debris disks around main-sequence stars ...

Dust plays an increasingly important role in astrophysics. It has a dramatic effect on the Universe by affecting the physical conditions and processes taking place within the Universe and shaping the appearance of dusty objects (e.g. cometary comae, reflection nebulae, dust disks, and galaxies) (i) as an absorber, scatterer, polarizer, and emitter of electromagnetic radiation; (ii) as a revealer of heavily obscured objects (e.g. IR sources) of which we might otherwise be unaware; (iii) as a driver for the mass loss of evolved stars; (iv) as a sink of heavy elements which if otherwise in the gas phase, would profoundly affect the interstellar gas chemistry; (v) as an efficient catalyst for the formation of $\mathrm{H}_{2}$ and other simple molecules as well as complex organic molecules (and as a protector by shielding them from photodissociating ultraviolet [UV] photons) in the interstellar medium (ISM); (vi) as an efficient 
agent for heating the interstellar gas by providing photoelectrons; (vii) as an important coolant in dense regions by radiating infrared (IR) photons (which is particularly important for the process of star formation in dense clouds by removing the gravitational energy of collapsing clouds and allowing star formation to take place); (viii) as an active participant in interstellar gas dynamics by communicating radiation pressure from starlight to the gas, and providing coupling of the magnetic field to the gas in regions of low fractional ionization; (ix) as a building block in the formation of stars and planetary bodies and finally, (x) as a diagnosis of the physical conditions (e.g. gas density, temperature, radiation intensity, electron density, magnetic field) of the regions where dust is seen.

The dust in the space between stars - interstellar dust - is the most extensively studied cosmic dust type, with circumstellar dust ("stardust"), cometary dust and IDPs coming second. Interstellar dust is an important constituent of the Milky Way and external galaxies. The presence of dust in galaxies limits our ability to interpret the local and distant Universe because dust extinction dims and reddens the galaxy light in the UV-optical-near-IR windows, where the vast majority of the astronomical data have been obtained. In order to infer the stellar content of a galaxy, or the history of star formation in the Universe, it is essential to correct for the effects of interstellar extinction. Dust absorbs starlight and reradiates at longer wavelengths. Nearly half of the bolometric luminosity of the local Universe is reprocessed by dust into the mid- and far-IR.

Stardust, cometary dust and IDPs are directly or indirectly related to interstellar dust: stardust, condensed in the cool atmospheres of evolved stars or supernova ejecta and subsequently injected into the ISM, is considered as a major source of interstellar dust, although the bulk of interstellar dust is not really stardust but must have recondensed in the ISM [11. Comets, because of their cold formation and cold storage, are considered as the most primitive objects in the solar system and best preserve the composition of the presolar molecular cloud among all solar system bodies. Greenberg [20] argued that comets are made of unaltered pristine interstellar materials with only the most volatile components partially evaporated, although it has also been proposed that cometary materials have been subjected to evaporation, recondensation and other reprocessing in the protosolar nebula and therefore have lost all the records of the presolar molecular cloud out of which they have formed. Genuine presolar grains have been identified in IDPs and primitive meteorites based on their isotopic anomalies [8, indicating that stardust can survive journeys from its birth in stellar outflows and supernova explosions, through the ISM, the formation of the solar system, and its ultimate incorporation into asteroids and comets.

Except in a few cases cosmic dust can be studied in situ (e.g. cometary dust 32, dust in the local interstellar cloud entering our solar system 22]) or in terrestrial laboratories (e.g. IDPs and meteorites [8], cometary dust [7]), our knowledge about cosmic dust is mainly derived from its interaction with 
electromagnetic radiation: extinction (scattering, absorption), polarization, and emission. Dust reveals its presence and physical and chemical properties and provides clues about the environment where it is found by scattering and absorbing starlight (or photons from other objects) and reradiating the absorbed energy at longer wavelengths.

This chapter deals with the optical properties of dust, i.e., how light is absorbed, scattered, and reradiated by cosmic dust. The subject of light scattering by small particles is a vast, fast-developing field. In this chapter I restrict myself to astrophysically-relevant topics. In $\$ 2$ I present a brief summary of the underlying physics of light scattering. The basic scattering terms are defined in 93 In 9 I discuss the physical basis of the dielectric functions of dust materials. In 95 and 86 I respectively summarize the analytic and numerical solutions for calculating the absorption and scattering parameters of dust.

\section{Scattering of Light by Dust: A Conceptual Overview}

When a dust grain, composed of discrete electric charges, is illuminated by an electromagnetic wave, the electric field of the incident electromagnetic wave will set the electric charges in the dust into oscillatory motion. These accelerated electric charges radiate electromagnetic energy in all directions, at the same frequency as that of the incident wave. This process, known as "scattering" (to be more precise, elastic scattering), removes energy from the incident beam of electromagnetic radiation. Absorption also arises as the excited charges transform part of the incident electromagnetic energy into thermal energy. The combined effect of absorption and scattering, known as "extinction", is the total energy loss of the incident wave [6.

The scattering of light by dust depends on the size, shape, and chemical composition of the dust and the direction at which the light is scattered. This can be qualitatively understood, as schematically shown in Figure 1 by conceptually subdividing the dust into many small regions, in each of which a dipole moment will be induced when illuminated by an incident electromagnetic wave. These dipoles oscillate at the frequency of the incident wave and therefore scatter secondary radiation in all directions. The total scattered field of a given direction (e.g. $P_{1}, P_{2}$ ) is the sum of the scattered wavelets, with their phase differences taken into account. Since these phase relations change for a different scattering direction, the scattered field varies with scattering direction 1 The phase relations among the scattered wavelets also change with the size and shape of the dust. On the other hand, the amplitude and phase of the induced dipole moment for a given frequency depend on the material of which the dust is composed. Therefore, the scattered field is sensitive to both the size, shape and chemical composition of the dust [6].

\footnotetext{
${ }^{1}$ An exception to this is the dust in the Rayleigh regime (i.e. with its size being much smaller than the wavelength) which scatters light nearly isotropically, with little variation with direction since for dust so small all the secondary wavelets are approximately in phase.
} 


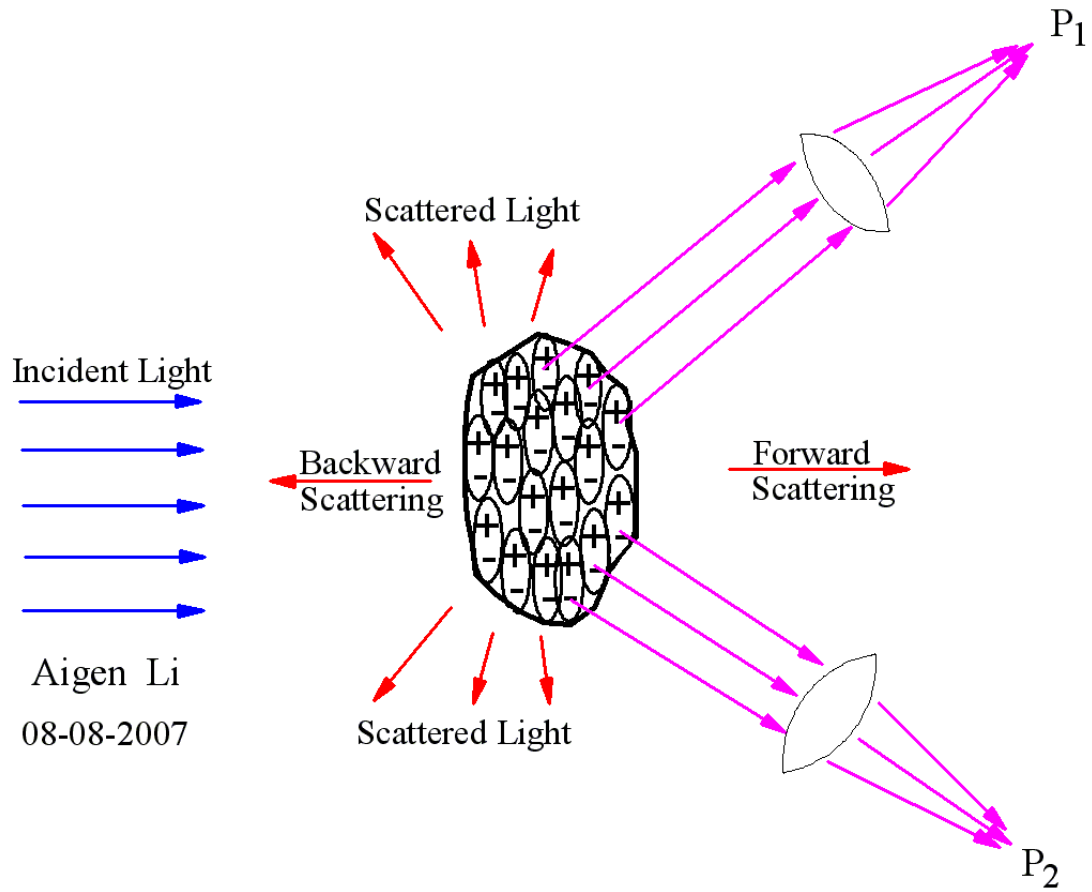

Fig. 1. A conceptual illustration of the scattering of light by dust. The dust is conceptually subdivided into many small regions. Upon illuminated by an incident electromagnetic wave, in each small region a dipole moment (of which the amplitude and phase depend on the composition the dust) will be induced. These dipoles oscillate at the frequency of the incident wave and scatter secondary radiation in all directions. The total scattered field of a given direction (e.g. $\left.P_{1}, P_{2}\right)$ is the sum of the scattered wavelets, where due account is taken of their phase differences. Since the phase relations among the scattered wavelets change with the scattering direction and the size and shape of the dust, the scattering of light by dust depends on the size, shape, and chemical composition of the dust and the direction at which the light is scattered.

\section{Scattering of Light by Dust: Definitions}

As discussed in $\$ 2$, when light impinges on a grain it is either scattered or absorbed. Let $I_{\mathrm{o}}(\lambda)$ be the intensity of the incident light at wavelength $\lambda$, the intensity of light scattered into a direction defined by $\theta$ and $\phi$ (see Fig.2) is

$$
I(\lambda)=\frac{I_{\mathrm{o}}(\lambda) F(\theta, \phi)}{k^{2} r^{2}}
$$

where $0^{\circ} \leq \theta \leq 180^{\circ}$ is the scattering angle (the angle from the incident direction), $0^{\circ} \leq \phi \leq 360^{\circ}$ is the azimuthal angle which uniquely determines the scattering plane defined by the incident direction and the scattering direc- 


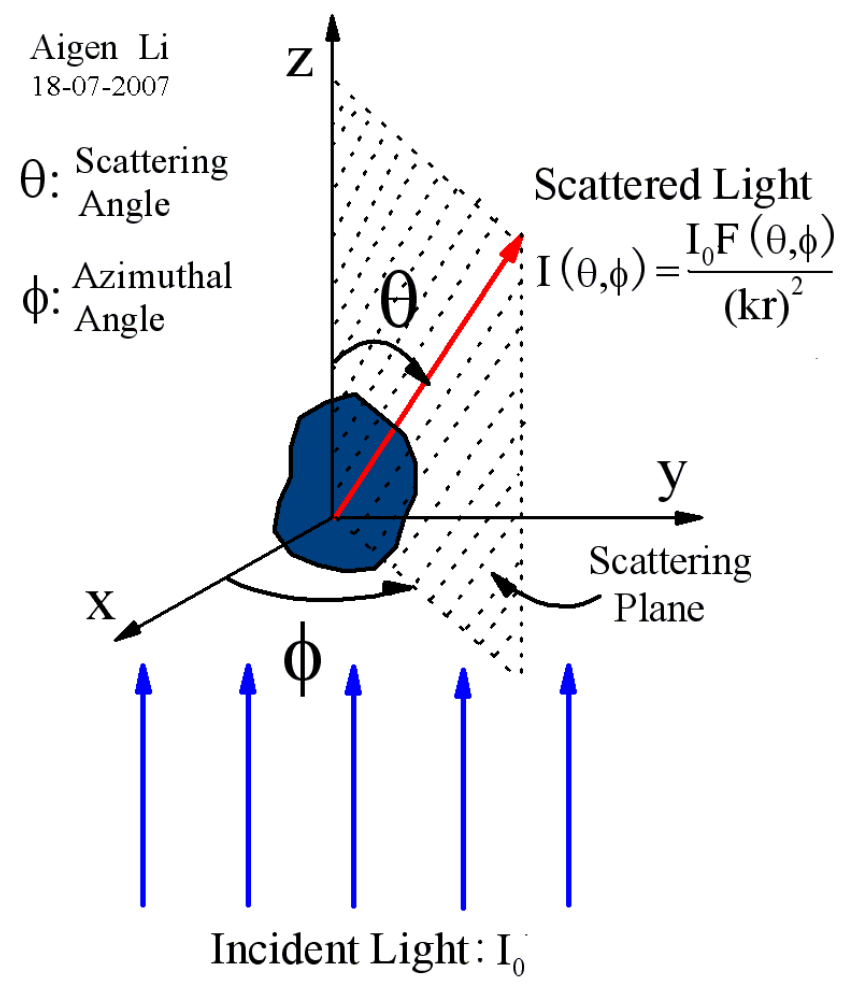

Fig. 2. Schematic scattering geometry of a dust grain in an incident radiation field of intensity $I_{\mathrm{o}}$ which scatters radiation of intensity $I(\theta, \phi)$ into a scattering angle $\theta\left(\theta=0^{\circ}\right.$ : forward scattering; $\theta=180^{\circ}$ : backward scattering), an azimuthal angle $\phi$, and a distance $r$ from the dust. In a Cartesian coordinate system, the incident direction defines the $+z$ axis. The scattering direction and the incident direction define the scattering plane. In the far-field region (i.e. $k r \gg 1), I=I_{\mathrm{o}} F(\theta, \phi) / k^{2} r^{2}$, where $k=2 \pi / \lambda$ is the wave number in vacuum.

tion (see Fig.22) $2_{2}^{2} F(\theta, \phi)$ is the (dimensionless) angular scattering function, $r \gg \lambda / 2 \pi$ is the distance from the scatterer, and $k=2 \pi / \lambda$ is the wave number in vacuum. The scattering cross section $C_{\text {sca }}$, defined as the area on which the incident wave falls with the same amount of energy as that scattered in all directions by the dust, may be obtained by integrating the angular scattering distribution $F(\theta, \phi) / k^{2}$ over all solid angles

$$
C_{\mathrm{sca}}(\lambda)=\frac{1}{k^{2}} \int_{0}^{2 \pi} \int_{0}^{\pi} F(\theta, \phi) \sin \theta d \theta d \phi
$$

${ }^{2}$ When the scattering is along the incident direction $\left(\theta=0^{\circ}\right.$, i.e. "forward scattering") or the scattering is on the opposite direction of the incident direction $\left(\theta=180^{\circ}\right.$, i.e. "backward scattering"), any plane containing the $z$ axis is a suitable scattering plane. 
where $F(\theta, \phi) / k^{2}$ (with a dimension of area) 3 after normalized by $C_{\text {sca }}$, is known as the phase function or scattering diagram

$$
p(\theta, \phi) \equiv \frac{F(\theta, \phi) / k^{2}}{C_{\text {sca }}} .
$$

The asymmetry parameter (or asymmetry factor) $g$ is defined as the average cosine of the scattering angle $\theta$

$g \equiv\langle\cos \theta\rangle=\int_{4 \pi} p(\theta, \phi) \cos \theta d \Omega=\frac{1}{k^{2} C_{\mathrm{sca}}} \int_{0}^{2 \pi} \int_{0}^{\pi} F(\theta, \phi) \cos \theta \sin \theta d \theta d \phi$.

The asymmetry parameter $g$, specifying the degree of scattering in the forward direction $\left(\theta=0^{\circ}\right)$, varies from -1 (i.e. all radiation is backward scattered like a "mirror") to 1 (for pure forward scattering). If a grain scatters more light toward the forward direction, $g>0 ; g<0$ if the scattering is directed more toward the back direction; $g=0$ if it scatters light isotropically (e.g. small grains in the Rayleigh regime) or if the scattering is symmetric with respect to $\theta=90^{\circ}$ (i.e. the scattered radiation is azimuthal independent and symmetric with respect to the plane perpendicular to the incident radiation).

In radiative transfer modeling of dusty regions, astronomers often use the empirical Henyey-Greenstein phase function to represent the anisotropic scattering properties of dust 26]

$$
H(\theta) \equiv \frac{1}{4 \pi} \frac{1-g^{2}}{\left(1+g^{2}-2 g \cos \theta\right)^{3 / 2}} .
$$

Draine (2003) proposed a more general analytic form for the phase function

$$
H_{\eta}(\theta) \equiv \frac{1}{4 \pi} \frac{3\left(1-g^{2}\right)}{3+\eta\left(1+2 g^{2}\right)} \frac{1+\eta \cos ^{2} \theta}{\left(1+g^{2}-2 g \cos \theta\right)^{3 / 2}}
$$

where $\eta$ is an adjustable parameter. For $\eta=0$ Eq 6 reduces to the HenyeyGreenstein phase function. For $g=0$ and $\eta=1 \mathrm{Eq}[6$ reduces to the phase function for Rayleigh scattering [12].

As discussed in $\$ 2$, both scattering and absorption (the sum of which is called extinction) remove energy from the incident beam. The extinction cross section, defined as

$$
C_{\text {ext }}=C_{\text {sca }}+C_{\text {abs }}=\frac{\text { total energy scattered and absorbed per unit time }}{\text { incident energy per unit area per unit time }},
$$

is determined from the optical theorem which relates $C_{\text {ext }}$ to the real part of the complex scattering amplitude $S(\theta, \phi)$ in the forward direction alone [28]

\footnotetext{
${ }^{3}$ Also called the "differential scattering cross section" $d C_{\text {sca }} / d \Omega \equiv F(\theta, \phi) / k^{2}$, it specifies the angular distribution of the scattered light [i.e. the amount of light (for unit incident irradiance) scattered into a unit solid angle about a given direction].

4 The angular scattering function $F(\theta, \phi)$ is just the absolute square of the complex scattering amplitude $S(\theta, \phi): F(\theta, \phi)=|S(\theta, \phi)|^{2}$.
} 


$$
\begin{aligned}
& \text { Optical Properties of Dust } \\
& C_{\text {ext }}=-\frac{4 \pi}{k^{2}} \operatorname{Re}\left\{S\left(\theta=0^{\circ}\right)\right\}
\end{aligned}
$$

The absorption cross section $C_{\text {abs }}$ is the area on which the incident wave falls with the same amount of energy as that absorbed inside the dust; $C_{\text {ext }}$, having a dimension of area, is the "effective" blocking area to the incident radiation (for grains much larger than the wavelength of the incident radiation, $C_{\text {ext }}$ is about twice the geometrical blocking area). For a grain (of size $a$ and complex index of refraction $m$ ) in the Rayleigh limit (i.e. $2 \pi a / \lambda \ll 1,2 \pi a|m| / \lambda \ll 1$ ), the absorption cross section $C_{\text {abs }}$ is much larger than the scattering cross section $C_{\text {sca }}$ and therefore $C_{\text {ext }} \approx C_{\text {abs }}$. Non-absorbing dust has $C_{\text {ext }}=C_{\text {sca }}$.

The albedo of a grain is defined as $\alpha \equiv C_{\mathrm{sca}} / C_{\text {ext }}$. For grains in the Rayleigh limit, $\alpha \approx 0$ since $C_{\text {sca }} \ll C_{\text {abs }}$. For Non-absorbing dust, $\alpha=1$.

In addition to energy, light carries momentum of which the direction is that of propagation and the amount is $h \nu / c$ (where $h$ is the Planck constant, $c$ is the speed of light, and $\nu$ is the frequency of the light). Therefore, upon illuminated by an incident beam of light, dust will acquire momentum and a force called radiation pressure will be exerted on it in the direction of propagation of the incident light. The radiation pressure force is proportional to the net loss of the forward component of the momentum of the incident beam. While the momentum of the absorbed light (which is in the forward direction) will all be transfered to the dust, the forward component of the momentum of the scattered light will not be removed from the incident beam. Therefore, the radiation pressure force exerted on the dust is

$$
F_{\mathrm{pr}}=I_{\mathrm{o}} C_{\mathrm{pr}} / c, C_{\mathrm{pr}}=C_{\mathrm{abs}}+(1-g) C_{\mathrm{sca}},
$$

where $C_{\mathrm{pr}}$ is the radiation pressure cross section, and $I_{\mathrm{O}}$ is the intensity (irradiance) of the incident light.

In literature, one often encounters $Q_{\text {ext }}, Q_{\text {sca }}, Q_{\text {abs }}$, and $Q_{\mathrm{pr}}$ - the extinction, scattering, absorption and radiation pressure efficiencies. They are defined as the extinction, scattering, absorption, and radiation pressure cross sections divided by the geometrical cross section of the dust $C_{\text {geo }}$,

$$
Q_{\mathrm{ext}}=\frac{C_{\mathrm{ext}}}{C_{\mathrm{geo}}} ; \quad Q_{\mathrm{sca}}=\frac{C_{\mathrm{sca}}}{C_{\mathrm{geo}}} ; \quad Q_{\mathrm{abs}}=\frac{C_{\mathrm{abs}}}{C_{\mathrm{geo}}} ; \quad Q_{\mathrm{pr}}=\frac{C_{\mathrm{pr}}}{C_{\mathrm{geo}}} .
$$

For spherical grains of radii $a, C_{\text {geo }}=\pi a^{2}$. For non-spherical grains, there is no uniformity in choosing $C_{\text {geo }}$. A reasonable choice is the geometrical cross

section of an "equal volume sphere" $C_{\text {geo }} \equiv \pi(3 V / 4 \pi)^{2 / 3} \approx 1.21 V^{2 / 3}$ where $V$ is the volume of the non-spherical dust.

\section{Scattering of Light by Dust: Dielectric Functions}

The light scattering properties of dust are usually evaluated based on its optical properties (i.e. dielectric functions or indices of refraction) and geometry (i.e. size and shape) by solving the Maxwell equations 


$$
\nabla \times \mathbf{E}+\frac{1}{c} \frac{\partial \mathbf{B}}{\partial t}=0 ; \nabla \times \mathbf{H}-\frac{1}{c} \frac{\partial \mathbf{D}}{\partial t}=\frac{4 \pi}{c} \mathbf{J} ; \nabla \cdot \mathbf{D}=4 \pi \rho ; \nabla \cdot \mathbf{B}=0 ;
$$

where $\mathbf{E}$ is the electric field, $\mathbf{B}$ is the magnetic induction, $\mathbf{H}$ is the magnetic field, $\mathbf{D}$ is the electric displacement, $\mathbf{J}$ is the electric current density, and $\rho$ is the electric charge density. They are supplemented with the constitutive relations (or "material relations")

$$
\mathbf{J}=\sigma \mathbf{E} ; \mathbf{D}=\varepsilon \mathbf{E}=(1+4 \pi \chi) \mathbf{E} ; \mathbf{B}=\mu \mathbf{H} ;
$$

where $\sigma$ is the electric conductivity, $\varepsilon=1+4 \pi \chi$ is the dielectric function (or permittivity or dielectric permeability), $\chi$ is the electric susceptibility, and $\mu$ is the magnetic permeability. For time-harmonic fields $\mathbf{E}, \mathbf{H} \propto \exp (-i \omega t)$, the Maxwell equations are reduced to the Helmholtz wave equations

$$
\nabla^{2} \mathbf{E}+\tilde{k}^{2} \mathbf{E}=0 ; \nabla^{2} \mathbf{H}+\tilde{k}^{2} \mathbf{H}=0 ;
$$

where $\tilde{k}=m \omega / c$ is the complex wavenumber, $m=\sqrt{\mu(\varepsilon+i 4 \pi \sigma / \omega)}$ is the complex refractive index, and $\omega=2 \pi c / \lambda$ is the circular frequency. These equations should be considered for the field outside the dust (which is the superposition of the incident field and the scattered field) and the field inside the dust, together with the boundary conditions (i.e. any tangential and normal components of $\mathbf{E}$ are continuous across the dust boundary). For non-magnetic dust $(\mu=1)$, the complex refractive index is $m=\sqrt{\varepsilon+i 4 \pi \sigma / \omega}$. For non-magnetic, non-conducting dust, $m=\sqrt{\varepsilon}$. For highly-conducting dust, $4 \pi \sigma / \omega \gg 1$, therefore, both the real part and the imaginary part of the index of refraction are approximately $\sqrt{2 \pi \sigma / \omega}$ at long wavelengths.

The complex refractive indices $m$ or dielectric functions $\varepsilon$ of dust are often called optical constants, although they are not constant but vary with wavelengths. They are of great importance in studying the absorption and scattering of light by dust. They are written in the form of $m=m^{\prime}+i m^{\prime \prime}$ and $\varepsilon=\varepsilon^{\prime}+i \varepsilon^{\prime \prime}$. The sign of the imaginary part of $m$ or $\varepsilon$ is opposite to that of the time-dependent term of the harmonically variable fields [i.e. $m^{\prime \prime}$, $\varepsilon^{\prime \prime}>0$ for $\mathbf{E}, \mathbf{H} \propto \exp (-i \omega t) ; m^{\prime \prime}, \varepsilon^{\prime \prime}<0$ for $\left.\mathbf{E}, \mathbf{H} \propto \exp (i \omega t)\right]$. The imaginary part of the index of refraction characterizes the attenuation of the wave $\left(4 \pi m^{\prime \prime} / \lambda\right.$ is called the absorption coefficient), while the real part determines the phase velocity $\left(\mathrm{c} / \mathrm{m}^{\prime}\right)$ of the wave in the medium: for an electric field propagating in an absorbing medium of $m=m^{\prime}+i m^{\prime \prime}$, say, in the $x$ direction, $\mathbf{E} \propto \exp \left(-\omega m^{\prime \prime} x / c\right) \exp \left[-i \omega\left(t-m^{\prime} x / c\right)\right]$.

The physical basis of the dielectric function $\varepsilon$ can readily be understood in terms of the classical Lorentz harmonic oscillator model (for insulators) and the Drude model (for free-electron metals). In the Lorentz oscillator model, the bound electrons and ions of a dust grain are treated as simple harmonic oscillators subject to the driving force of an applied electromagnetic field. The applied field distorts the charge distribution and therefore produces an induced dipole moment. To estimate the induced moments we consider a (polarizable) grain as a collection of identical, independent, isotropic, harmonic oscillators 
with mass $m$ and charge $q$; each oscillator is under the action of three forces: (i) a restoring force $-K \mathbf{x}$, where $K$ is the force constant (i.e. stiffness) of the "spring" to which the bound charges are attached, and $\mathbf{x}$ is the displacement of the bound charges from their equilibrium; (ii) a damping force $-b \dot{\mathbf{x}}$, where $b$ is the damping constant; and (iii) a driving force $q \mathbf{E}$ produced by the (local) electric field $\mathbf{E}$. The equation of motion of the oscillators is

$$
m \ddot{\mathbf{x}}+b \dot{\mathbf{x}}+K \mathbf{x}=q \mathbf{E} .
$$

For time harmonic electric fields $\mathbf{E} \propto \exp (-i \omega t)$, we solve for the displacement

$$
\mathbf{x}=\frac{(q / m) \mathbf{E}}{\omega_{\mathrm{o}}^{2}-\omega^{2}-i \gamma \omega}, \omega_{\mathrm{o}}^{2}=K / m, \gamma=b / m
$$

where $\omega_{0}$ is the frequency of oscillation about equilibrium (i.e. the restoring force is $\left.-m \omega_{\mathrm{o}}^{2} \mathbf{x}\right)$. The induced dipole moment $\mathbf{p}$ of an oscillator is $\mathbf{p}=q \mathbf{x}$. Let $n$ be the number of oscillators per unit volume. The polarization (i.e. dipole moment per unit volume) $\mathbf{P}=n \mathbf{p}=n q \mathbf{x}$ is

$$
\mathbf{P}=\frac{1}{4 \pi} \frac{\omega_{\mathrm{p}}^{2}}{\omega_{\mathrm{o}}^{2}-\omega^{2}-i \gamma \omega} \mathbf{E}, \quad \omega_{\mathrm{p}}^{2}=4 \pi n q^{2} / m
$$

where $\omega_{\mathrm{p}}$ is the plasma frequency. Since $\mathbf{P}=\chi \mathbf{E}=(\varepsilon-1) / 4 \pi \mathbf{E}$, the dielectric function for a one-oscillator model around a resonance frequency $\omega_{\mathrm{o}}$ is

$$
\begin{gathered}
\varepsilon=1+4 \pi \chi=1+\frac{\omega_{\mathrm{p}}^{2}}{\omega_{\mathrm{o}}^{2}-\omega^{2}-i \gamma \omega} \\
\varepsilon^{\prime}=1+4 \pi \chi^{\prime}=1+\frac{\omega_{\mathrm{p}}^{2}\left(\omega_{\mathrm{o}}^{2}-\omega^{2}\right)}{\left(\omega_{\mathrm{o}}^{2}-\omega^{2}\right)^{2}+\gamma^{2} \omega^{2}} \\
\varepsilon^{\prime \prime}=4 \pi \chi^{\prime \prime}=\frac{\omega_{\mathrm{p}}^{2} \gamma \omega}{\left(\omega_{\mathrm{o}}^{2}-\omega^{2}\right)^{2}+\gamma^{2} \omega^{2}} .
\end{gathered}
$$

We see from $\mathrm{Eq} 19 \varepsilon^{\prime \prime} \geq 0$ for all frequencies. This is true for materials close to thermodynamic equilibrium, except those with population inversions. If there are many oscillators of different frequencies, the dielectric function for a multiple-oscillator model is

$$
\varepsilon=1+\sum_{j} \frac{\omega_{\mathrm{p}, j}^{2}}{\omega_{j}^{2}-\omega^{2}-i \gamma_{j} \omega}
$$

where $\omega_{\mathrm{p}, j}, \omega_{j}$ and $\gamma_{j}$ are respectively the plasma frequency, the resonant frequency, and the damping constant of the $j$-th oscillator.

The optical properties associated with free electrons are described by the Drude model. The free electrons experience no restoring forces when driven by the electric field of a light wave and do not have natural resonant frequencies 


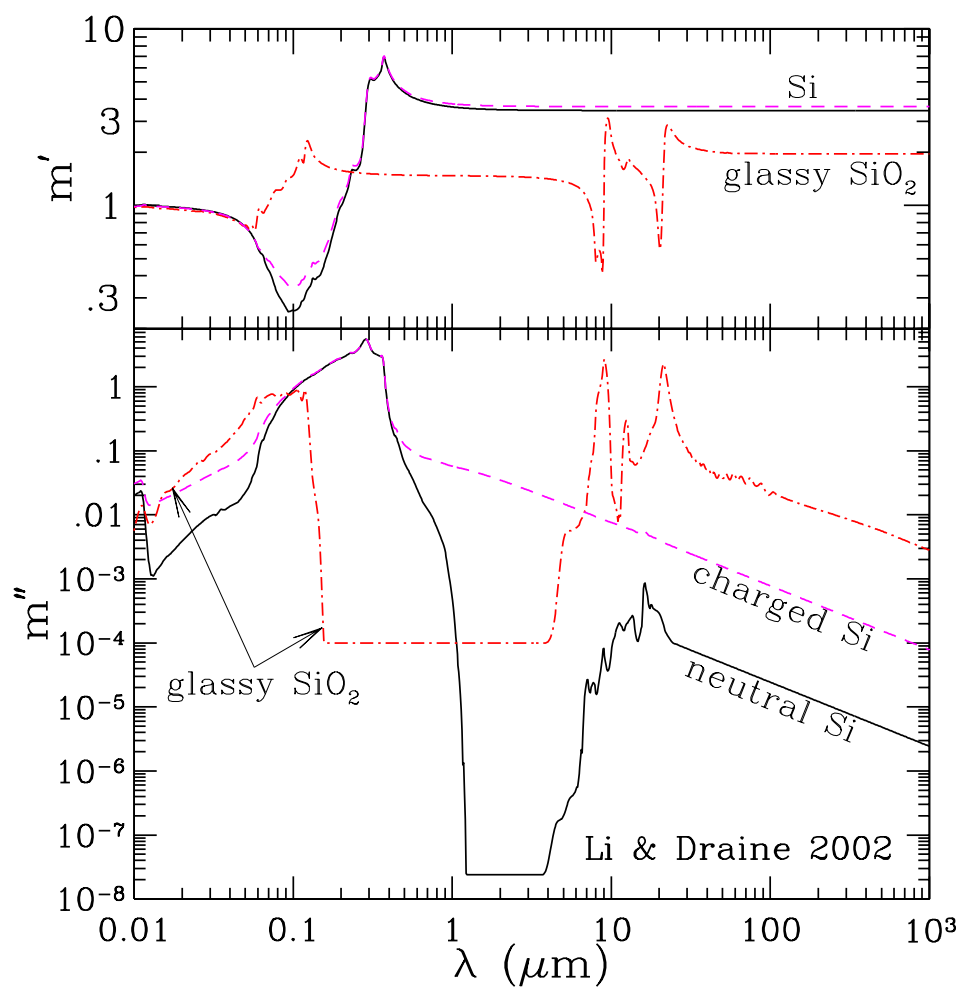

Fig. 3. Refractive indices $m^{\prime}$ (upper panel), $m^{\prime \prime}$ (lower panel) of neutral silicon nanoparticles ( $\mathrm{Si}$; solid lines), singly, positively charged $(Z=+1)$ silicon nanoparticles of size $a=10 \AA$ (dashed lines), and $\mathrm{SiO}_{2}$ glass (dot-dashed lines). Compared to neutral $\mathrm{Si}$, in charged $\mathrm{Si}$ free electrons (if negative charged) or holes (if positively charged) contribute to the dielectric function. Crystalline $\mathrm{Si}$ is IR inactive since its lattice vibrations have no dipole moment; the bands at $6.91,7.03,7.68,8.9,11.2$, $13.5,14.5,16.4$, and $17.9 \mu \mathrm{m}$ are due to multi-phonon processes. Taken from 48 .

(i.e. $\omega_{\mathrm{o}}=0$ ). The Drude model for metals is obtained directly from the Lorentz model for insulators simply by setting the restoring force in Eq14 equal to zero. The dielectric function for free electrons is

$$
\begin{gathered}
\varepsilon=1-\frac{\omega_{\mathrm{p}, e}^{2}}{\omega^{2}+i \gamma_{e} \omega}, \omega_{\mathrm{p}, e^{2}}=4 \pi n_{e} e^{2} / m_{e} \\
\varepsilon^{\prime}=1-\frac{\omega_{\mathrm{p}, e^{2}}}{\omega^{2}+\gamma_{e}^{2}} \\
\varepsilon^{\prime \prime}=\frac{\omega_{\mathrm{p}, e^{2} \gamma_{e}}}{\omega\left(\omega^{2}+\gamma_{e}^{2}\right)}
\end{gathered}
$$

where $\omega_{\mathrm{p}, e}$ is the plasma frequency, $n_{e}$ is the density of free electrons and $m_{e}$ is the effective mass of an electron. The damping constant $\gamma_{e}=1 / \tau_{e}$ is 
the reciprocal of the mean free time between collisions $\left(\tau_{e}\right)$ which are often determined by electron-phonon scattering (i.e. interaction of the electrons with lattice vibrations) 5 For dust materials (e.g. graphite) containing both bound charges and free electrons, the dielectric function is

$$
\varepsilon=1-\frac{\omega_{\mathrm{p}, e} e^{2}}{\omega^{2}+i \gamma_{e} \omega}+\sum_{j} \frac{\omega_{\mathrm{p}, j}^{2}}{\omega_{j}^{2}-\omega^{2}-i \gamma_{j} \omega} .
$$

The optical response of free electrons in metals can also be understood in terms of the electric current density $\mathbf{J}$ and conductivity $\sigma(\omega)$. The free electrons in metals move between molecules. In the absence of an external field, they move in a random manner and hence they do not give rise to a net current flow. When an external field is applied, the free electrons acquire an additional velocity and their motion becomes more orderly which gives rise to an induced current flow. The current density $\mathbf{J}=-n_{e} e \dot{\mathbf{x}}$ is obtained by solving the equation of motion of the free electrons $m_{e} \ddot{\mathbf{x}}=-e \mathbf{E}-m_{e} \dot{\mathbf{x}} / \tau_{e}$

$$
\dot{\mathbf{x}}=\frac{-\tau_{e} e}{m_{e}} \frac{1}{1-i \omega \tau_{e}} \mathbf{E}
$$

Since $\mathbf{J}=\sigma \mathbf{E}$, the a.c. conductivity is $\sigma=\sigma_{\mathrm{o}} /\left(1-i \omega \tau_{e}\right)$, where $\sigma_{\mathrm{o}}=$ $n_{e} \tau_{e} e^{2} / m_{e}$ is the d.c. conductivity. The dielectric function for a free-electron metal is therefore

$$
\varepsilon=1+\frac{i 4 \pi \sigma}{\omega}=1-\frac{\omega_{\mathrm{p}, e}^{2}}{\omega^{2}+i \omega / \tau_{e}} .
$$

The real and imaginary parts of the dielectric function are not independent. They are related through the Kramers-Kronig (or dispersion) relations

$$
\varepsilon^{\prime}(\omega)=1+\frac{2}{\pi} P \int_{0}^{\infty} \frac{x \varepsilon^{\prime \prime}(x)}{x^{2}-\omega^{2}} d x \quad, \quad \varepsilon^{\prime \prime}(\omega)=\frac{-2 \omega}{\pi} P \int_{0}^{\infty} \frac{\varepsilon^{\prime}(x)}{x^{2}-\omega^{2}} d x
$$

where $P$ is the Cauchy Principal value of the integral

\footnotetext{
${ }^{5}$ For nano-sized metallic dust of size $a$ (which is smaller than the mean free path of conduction electrons in the bulk metal), $\tau_{e}$ and $\gamma_{e}$ are increased because of additional collisions with the boundary of the dust: $\gamma_{e}=\gamma_{\mathrm{bulk}}+v_{F} /(\varsigma a)$, where $\gamma_{\mathrm{bulk}}$ is the bulk metal damping constant, $v_{F}$ is the electron velocity at the Fermi surface, $\varsigma$ is a dimensionless constant of order unity which depends on the character of the scattering at the boundary ( $\varsigma a$ is the effective mean free path for collisions with the boundary): $\varsigma=1$ for classic isotropic scattering, $\varsigma=4 / 3$ for classic diffusive scattering, $\varsigma=1.16$ or $\varsigma=1.33$ for scattering based on the quantum particle-ina-box model (see 9] and references therein). Since $\omega^{2} \gg \gamma_{e}^{2}$ in metals near the plasma frequency, $\varepsilon^{\prime \prime}$ can be written as $\varepsilon^{\prime \prime}=\varepsilon^{\prime \prime}$ bulk $+v_{F} \omega_{\mathrm{p}}{ }^{2} /\left(\varsigma a \omega^{3}\right)$. This, known as the "electron mean free path limitation" effect, indicates that for a metallic grain $\varepsilon^{\prime \prime}$ increases as the grain becomes smaller.
} 


$$
P \int_{0}^{\infty} \frac{x \varepsilon^{\prime \prime}(x)}{x^{2}-\omega^{2}} d x=\lim _{a \rightarrow 0}\left[\int_{0}^{\omega-a} \frac{x \varepsilon^{\prime \prime}(x)}{x^{2}-\omega^{2}} d x+\int_{\omega+a}^{\infty} \frac{x \varepsilon^{\prime \prime}(x)}{x^{2}-\omega^{2}} d x\right] .
$$

The real and imaginary parts of the index of refraction are also connected through the Kramers-Kronig relation. This also holds for the real and imaginary parts of the electric susceptibility.

$$
\begin{gathered}
m^{\prime}(\omega)=1+\frac{2}{\pi} P \int_{0}^{\infty} \frac{x m^{\prime \prime}(x)}{x^{2}-\omega^{2}} d x, \quad m^{\prime \prime}(\omega)=\frac{-2 \omega}{\pi} P \int_{0}^{\infty} \frac{m^{\prime}(x)}{x^{2}-\omega^{2}} d x \\
\chi^{\prime}(\omega)=\frac{2}{\pi} P \int_{0}^{\infty} \frac{x \chi^{\prime \prime}(x)}{x^{2}-\omega^{2}} d x, \quad \chi^{\prime \prime}(\omega)=\frac{-2 \omega}{\pi} P \int_{0}^{\infty} \frac{\chi^{\prime}(x)}{x^{2}-\omega^{2}} d x
\end{gathered}
$$

The Kramers-Kronig relation can be used to relate the extinction cross section integrated over the entire wavelength range to the dust volume $V$

$$
\int_{0}^{\infty} C_{\mathrm{ext}}(\lambda) d \lambda=3 \pi^{2} F V
$$

where $F$, a dimensionless factor, is the orientationally-averaged polarizability relative to the polarizability of an equal-volume conducting sphere, depending only upon the grain shape and the static (zero-frequency) dielectric constant $\epsilon_{\mathrm{o}}$ of the grain material [64]. This has also been used to place constraints on interstellar grain models based on the interstellar depletions [46, 50] and the carrier of the mysterious $21 \mu \mathrm{m}$ emission feature seen in 12 protoplanetary nebulae 44 .

For illustration, we show in Figure 3 the refractive indices of glassy $\mathrm{SiO}_{2}$ and neutral and singly-charged silicon nanoparticles (SNPs) 6 The optical properties of SNPs depend on whether any free electrons or holes are present. The contribution of free electrons or holes to the dielectric function of SNPs is approximated by $\delta \varepsilon \approx-\omega_{\mathrm{p}}{ }^{2} \tau^{2} /\left(\omega^{2} \tau^{2}+i \omega \tau\right), \omega_{\mathrm{p}}{ }^{2}=3|Z| e^{2} / a^{3} m_{\mathrm{eff}}$, where $e$ is the proton charge, $Z e$ is the grain charge, $a$ is the grain radius, $\tau \approx a / v_{F}$ is the collision time (we take $v_{F} \approx 10^{8} \mathrm{~cm} \mathrm{~s}^{-1}$ ), and $m_{\mathrm{eff}}$ is the effective mass of a free electron or hole. In Figure 3 the charged SNPs are taken to contain only one hole (i.e. $Z=+1$ ). We take $m_{\text {eff }} \approx 0.2 m_{e}$.

\section{Scattering of Light by Dust: Analytic Solutions}

For dust with sizes much smaller than the wavelength of the incident radiation, analytic solutions to the light scattering problem exist for certain shapes. Let $a$ be the characteristic length of the dust, and $x \equiv 2 \pi a / \lambda$ be the dimensionless size parameter. Under the condition of $x \ll 1$ and $|m x| \ll 1$ (i.e. in the "Rayleigh" regime), $C_{\text {abs }}=4 \pi k \operatorname{Im}\{\boldsymbol{\alpha}\}, C_{\text {sca }}=(8 \pi / 3) k^{4}|\boldsymbol{\alpha}|^{2}$, where $\boldsymbol{\alpha}$ is

${ }^{6}$ SNPs were proposed as the carrier of the "extended red emission" (ERE), a broad, featureless emission band between $\sim 5400$ and $9000 \AA$ seen in a wide variety of dusty environments [72, 41] (but see [48]). SNPs were also suggested to be present in the inner corona of the Sun [24] (but see [54, 55]). 
the complex electric polarizability of the dust. Apparently, $C_{\mathrm{sca}} \ll C_{\mathrm{abs}}$ and $C_{\text {ext }} \approx C_{\text {abs. }}$. In general, $\boldsymbol{\alpha}$ is a diagonalized tensor 7 for homogeneous spheres composed of an isotropic material, it is independent of direction

$$
\alpha_{j j}=\frac{3 V}{4 \pi} \frac{\varepsilon-1}{\varepsilon+2},
$$

where $V$ is the dust volume 8 For a homogeneous, isotropic ellipsoid, the polarizability for electric field vector parallel to its principal axis $j$ is

$$
\alpha_{j j}=\frac{V}{4 \pi} \frac{\varepsilon-1}{(\varepsilon-1) L_{j}+1},
$$

where $L_{j}$ is the "depolarization factor" along principal axis $j$ (see [13). The electric polarizability $\boldsymbol{\alpha}$ is also known for concentric core-mantle spheres 69, confocal core-mantle ellipsoids [13, 19, and multi-layered ellipsoids of equaleccentricity [18. For a thin conducting cylindrical rod with length $2 l$ and radius $r_{a} \ll l$, the polarizability along the axis of the rod is [38]

$$
\alpha_{j j} \approx \frac{l^{3}}{3 \log \left(4 l / r_{a}\right)-7} .
$$

In astronomical modeling, the most commonly invoked grain shapes are spheres and spheroids (oblates or prolates) 9 In the Rayleigh regime, their absorption and scattering properties are readily obtained from Eqs.(32)35). For both dielectric and conducting spheres (as long as $x \ll 1$ and $|m x| \ll 1$ )

$$
C_{\text {abs }} / V=\frac{9 \omega}{c} \frac{\varepsilon^{\prime \prime}}{\left(\varepsilon^{\prime}+2\right)^{2}+\varepsilon^{\prime \prime 2}} \gg C_{\text {sca }} / V=\frac{3}{2 \pi}\left(\frac{\omega}{c}\right)^{4}\left|\frac{\varepsilon-1}{\varepsilon+2}\right|^{2} .
$$

${ }^{7} \boldsymbol{\alpha}$ can be diagonalized by appropriate choice of Cartesian coordinate system. It describes the linear response of a dust grain to applied electric field $\mathbf{E}: \mathbf{p}=\boldsymbol{\alpha} \mathbf{E}$ where $\mathbf{p}$ is the induced electric dipole moment.

${ }^{8}$ For a dielectric sphere with dielectric function given in Eq.(17), in the Rayleigh regime the absorption cross section is

$$
C_{\text {abs }}(\omega) / V=\frac{9}{c} \frac{\gamma \omega_{\mathrm{p}}^{2} \omega^{2}}{\left(3 \omega^{2}-\omega_{\mathrm{p}}^{2}-3 \omega_{\mathrm{o}}^{2}\right)^{2}+9 \gamma^{2} \omega^{2}} .
$$

Similarly, for a metallic sphere with dielectric function given in Eq. (21),

$$
C_{\mathrm{abs}}(\omega) / V=\frac{9}{c} \frac{\gamma_{e} \omega_{\mathrm{p}, e^{2} \omega^{2}}}{\left(3 \omega^{2}-\omega_{\mathrm{p}, e^{2}}\right)^{2}+9 \gamma_{e}^{2} \omega^{2}} .
$$

It is seen that the frequency-dependent absorption cross section for both dielectric and metallic spheres is a Drude function. This is also true for ellipsoids.

${ }^{9}$ Spheroids are a special class of ellipsoids. Let $r_{a}, r_{b}$, and $r_{c}$ be the semi-axes of an ellipsoid. For spheroids, $r_{b}=r_{c}$. Prolates with $r_{a}>r_{b}$ are generated by rotating an ellipse (of semi-major axis $r_{a}$ and semi-minor axis $r_{b}$ ) about its major axis; oblates with $r_{a}<r_{b}$ are generated by rotating an ellipse (of semi-minor axis $r_{a}$ and semi-major axis $r_{b}$ ) about its minor axis. 
At long wavelengths, for dielectric dust $\varepsilon^{\prime \prime} \propto \omega$ while $\varepsilon^{\prime}$ approaches a constant much larger than $\varepsilon^{\prime \prime}$ (see Eqs 1819), we see $C_{\text {abs }} \propto \omega \varepsilon^{\prime \prime} \propto \omega^{2}$; for metallic dust, $\varepsilon^{\prime \prime} \propto 1 / \omega$ while $\varepsilon^{\prime}$ approaches a constant much smaller than $\varepsilon^{\prime \prime}$ (see Eqs 22231), we see $C_{\text {abs }} \propto \omega / \varepsilon^{\prime \prime} \propto \omega^{2}$; therefore, for both dielectric and metallic dust $C_{\text {abs }} \propto \lambda^{-2}$ at long wavelengths 10

It is also seen from Eq.(37) that for spherical dust in the Rayleigh regime the albedo $\alpha \approx 0$, and the radiation cross section $C_{\mathrm{pr}} \approx C_{\mathrm{abs}}$. This has an interesting implication. Let $\beta_{\mathrm{pr}}(a)$ be the ratio of the radiation pressure force to the gravity of a spherical grain of radius $a$ in the solar system or in debris disks illuminated by stars (of radius $R_{\star}$ and mass $M_{\star}$ ) with a stellar flux of $F_{\lambda}^{\star}$ at the top of the atmosphere [53],

$$
\beta_{\mathrm{pr}}(a)=\frac{3 R_{\star}^{2} \int F_{\lambda}^{\star}\left[C_{\mathrm{abs}}(a, \lambda)+(1-g) C_{\mathrm{sca}}(a, \lambda)\right] d \lambda}{16 \pi c G M_{\star} a^{3} \rho_{\text {dust }}}
$$

where $G$ is the gravitational constant, and $\rho_{\text {dust }}$ is the mass density of the dust. For grains in the Rayleigh regime $\left(g \approx 0 ; C_{\mathrm{sca}} \ll C_{\text {abs }} ; C_{\text {abs }} \propto a^{3}\right)$, we see $\beta_{\mathrm{pr}} \propto C_{\mathrm{abs}} / a^{3}$ is independent of the grain size $a$ (see Fig.(4)!

Spheroids are often invoked to model the interstellar polarization. In the Rayleigh approximation, their absorption cross sections for light polarized parallel $(\|)$ or perpendicular $(\perp)$ to the grain symmetry axis are11

$$
C_{\text {abs }}^{\|, \perp} / V=\frac{\omega}{c} \operatorname{Im}\left\{\frac{\varepsilon-1}{(\varepsilon-1) L_{\|, \perp}+1}\right\},
$$

where the depolarization factors parallel $\left(L_{\|}\right)$or perpendicular $\left(L_{\perp}\right)$ to the grain symmetry axis are not independent, but related to each other through $L_{\|}+2 L_{\perp}=1$, with

$$
L_{\|}=\frac{1-\xi_{e}^{2}}{\xi_{e}^{2}}\left[\frac{1}{2 \xi_{e}} \ln \left(\frac{1+\xi_{e}}{1-\xi_{e}}\right)-1\right] \quad, \quad \xi_{e}=\sqrt{1-\left(r_{b} / r_{a}\right)^{2}}
$$

${ }^{10}$ However, various astronomical data suggest a flatter wavelength-dependence (i.e. $C_{\text {abs }} \propto \lambda^{-\beta}$ with $\left.\beta<2\right): \beta<2$ in the far-IR/submm wavelength range has been reported for interstellar molecular clouds, circumstellar disks around young stars, and circumstellar envelopes around evolved stars. Laboratory measurements have also found $\beta<2$ for certain cosmic dust analogues. In literature, the flatter $(\beta<2)$ long-wavelength opacity law is commonly attributed to grain growth by coagulation of small dust into large fluffy aggregates (see 47] and references therein). However, as shown in Eq. (31), the Kramers-Kronig relation requires that $\beta$ should be larger than 1 for $\lambda \rightarrow \infty$ since $F$ is a finite number and the integration in the left-hand-side of Eq. (31) should be convergent although we cannot rule out $\beta<1$ over a finite range of wavelengths.

${ }^{11}$ For grains spinning around the principal axis of the largest moment of inertia, the polarization cross sections are $C_{\mathrm{pol}}=\left(C_{\mathrm{abs}}^{\|}-C_{\mathrm{abs}}^{\perp}\right) / 2$ for prolates, and $C_{\mathrm{pol}}=$ $\left(C_{\mathrm{abs}}^{\perp}-C_{\mathrm{abs}}^{\|}\right)$for oblates; the absorption cross sections for randomly-oriented spheroids are $C_{\mathrm{abs}}=\left(C_{\mathrm{abs}}^{\|}+2 C_{\mathrm{abs}}^{\perp}\right) / 3[42$. 


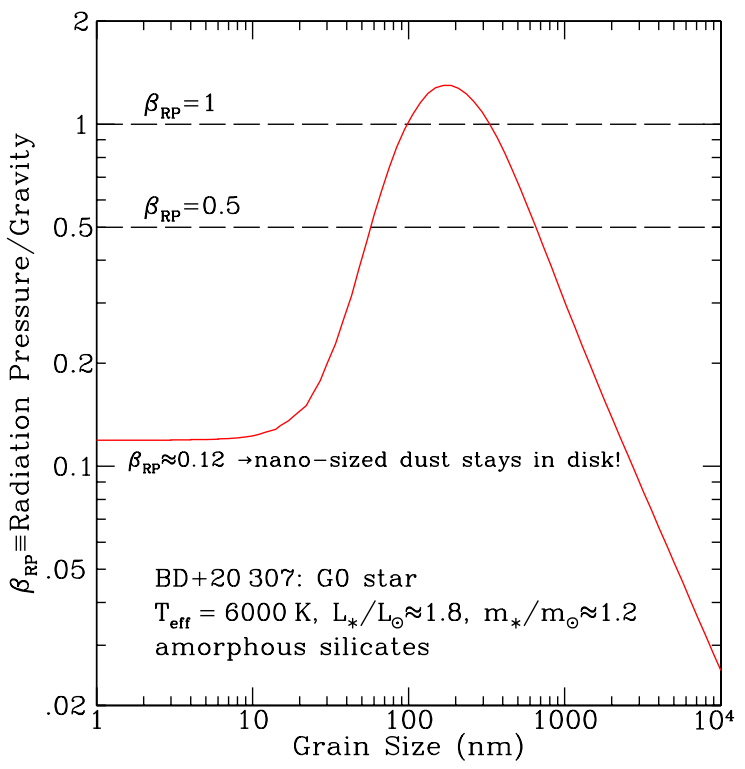

Fig. 4. $\beta_{\mathrm{pr}}-$ Ratio of radiation pressure to gravity for compact silicate grains in the debris disk around the Sun-like star BD+20 307 (G0, age $\sim 300 \mathrm{Myr}$ ). We note for nano-sized grains $\beta_{\mathrm{pr}} \approx 0.12$, independent of grain size; for grains larger than $\sim 0.3 \mu \mathrm{m}, \beta_{\mathrm{pr}}$ is inverse proportional to grain size. Song et al. 68 attributed the dust in this disk to recent extreme collisions between asteroids. Taken from 51.

for prolates $\left(r_{a}>r_{b}\right)$ where $\xi_{e}$ is the eccentricity, and

$$
L_{\|}=\frac{1+\xi_{e}^{2}}{\xi_{e}^{2}}\left(1-\frac{1}{\xi_{e}} \arctan \xi_{e}\right) \quad, \quad \xi_{e}=\sqrt{\left(r_{b} / r_{a}\right)^{2}-1}
$$

for oblates $\left(r_{a}<r_{b}\right)$. For spheres $L_{\|}=L_{\perp}=1 / 3$ and $\xi_{e}=0$. For extremely elongated prolates or "needles" $\left(r_{a} \gg r_{b}\right)$, it is apparent $C_{\mathrm{abs}}^{\perp} \ll C_{\mathrm{abs}}^{\|}$, we thus obtain

$$
C_{\mathrm{abs}} / V \approx \frac{\omega}{3 c} \frac{\varepsilon^{\prime \prime}}{\left[L_{\|}\left(\varepsilon^{\prime}-1\right)+1\right]^{2}+\left(L_{\|} \varepsilon^{\prime \prime}\right)^{2}}
$$

where $L_{\|} \approx\left(r_{b} / r_{a}\right)^{2} \ln \left(r_{a} / r_{b}\right)$. For dielectric needles, $C_{\mathrm{abs}} \propto \omega \varepsilon^{\prime \prime} \propto \lambda^{-2}$ at long wavelengths since $L_{\|}\left(\varepsilon^{\prime}-1\right)+1 \gg L_{\|} \varepsilon^{\prime \prime}$ (see [45]); for metallic needles, for a given value of $\varepsilon^{\prime \prime}$ one can always find a sufficiently long needle with $L_{\|} \varepsilon^{\prime \prime}<1$ and $L_{\|}\left(\varepsilon^{\prime}-1\right) \ll 1$ so that $C_{\text {abs }} \propto \omega \varepsilon^{\prime \prime} \propto \sigma$ which can be very large (see [45]). Because of their unique optical properties, metallic needles with high electrical conductivities (e.g. iron needles, graphite whiskers) are resorted to explain a wide variety of astrophysical phenomena: (1) as a source of starlight opacity to create a non-cosmological microwave background by the thermalization of starlight in a steady-state cosmology [27]; (2) as a source of 
the grey opacity needed to explain the observed redshift-magnitude relation of Type Ia supernovae without invoking a positive cosmological constant 11; (3) as the source for the submm excess observed in the Cas A supernova remnant [16; and (4) as an explanation for the flat 3-8 $\mu \mathrm{m}$ extinction observed for lines of sight toward the Galactic Center and in the Galactic plane 15. However, caution should be taken in using Eq42 (i.e. the Rayleigh approximation) since the Rayleigh criterion $2 \pi r_{a}|m| / \lambda \ll 1$ is often not satisfied for highly conducting needles (see [45) 12

In astronomical spectroscopy modeling, the continuous distribution of ellipsoid (CDE) shapes has been widely used to approximate the spectra of irregular dust grains by averaging over all ellipsoidal shape parameters [6]. In the Rayleigh limit, this approach, assuming that all ellipsoidal shapes are equally probable, has a simple expression for the average cross section

$$
\left\langle C_{\text {abs }} / V\right\rangle=\frac{\omega}{c} \operatorname{Im}\left\{\frac{2 \varepsilon}{\varepsilon-1} \log \varepsilon\right\}, \log \varepsilon \equiv \ln \sqrt{{\varepsilon^{\prime}}^{2}+\varepsilon^{\prime \prime 2}}+i \arctan \left(\varepsilon^{\prime \prime} / \varepsilon^{\prime}\right)
$$

where $\log \varepsilon$ is the principal value of the $\operatorname{logarithm}$ of $\varepsilon$. The CDE approach, resulting in a significantly-broadened spectral band (but with its maximum reduced), seems to fit the experimental absorption spectra of solids better than Mie theory. Although the CDE may indeed represent a distribution of shape factors caused either by highly irregular dust shapes or by clustering of spherical grains into irregular agglomerates, one should caution that the shape distribution of cosmic dust does not seem likely to resemble the CDE, which assumes that extreme shapes like needles and disks are equally probable. A more reasonable shape distribution function would be like $d P / d L_{\|}=12 L_{\|}\left(1-L_{\|}\right)^{2}$ which peaks at spheres $\left(L^{\|}=1 / 3\right)$. This function is symmetric about spheres with respect to eccentricity $e$ and drops to zero for the extreme cases: infinitely thin needles $\left(e \rightarrow 1, L_{\|} \rightarrow 0\right)$ or infinitely flattened pancakes $\left(e \rightarrow \infty, L_{\|} \rightarrow 1\right)$. Averaging over the shape distribution, the resultant absorption cross section is $C_{\text {abs }}=\int_{0}^{1} d L_{\|} d P / d L_{\|} C_{\text {abs }}\left(L_{\|}\right)$where $C_{\text {abs }}\left(L_{\|}\right)$is the absorption cross section of a particular shape $L_{\|}$62, 49, 48, Alternatively, Fabian et al. [17, proposed a quadratic weighting for the shape distribution, "with near-spherical shapes being most probable".

When a dust grain is very large compared with the wavelength, the electromagnetic radiation may be treated by geometric optics: $Q_{\text {ext }} \equiv C_{\text {ext }} / C_{\text {geo }} \rightarrow 2$

\footnotetext{
12 The "antenna theory" has been applied for conducting needle-like dust to estimate its absorption cross sections [75]. Let it be represented by a circular cylinder of radius $r_{a}$ and length $l\left(r_{a} \ll l\right)$. Let $\rho_{\mathrm{R}}$ be its resistivity. The absorption cross section is given by $C_{\text {abs }}=(4 \pi / 3 c)\left(\pi r_{a}^{2} l / \rho_{\mathrm{R}}\right)$, with a long wavelength cutoff of $\lambda_{\mathrm{o}}=$ $\rho_{\mathrm{R}} c\left(l / r_{a}\right)^{2} / \ln \left(l / r_{a}\right)^{2}$, and a short-wavelength cutoff of $\lambda_{\min } \approx\left(2 \pi c m_{e}\right) /\left(\rho_{\mathrm{R}} n_{e} e^{2}\right)$, where $m_{e}, e$, and $n_{e}$ are respectively the mass, charge, and number density of the charge-carrying electrons.
} 
if $x \equiv 2 \pi a / \lambda \gg 1$ and $|m-1| x \gg 113$ For these grains $\left(g \approx 1 ; C_{\text {abs }} \approx C_{\text {geo }}\right)$, the ratio of the radiation pressure to gravity $\beta_{\mathrm{pr}} \propto C_{\mathrm{abs}} / a^{3} \propto 1 / a$. This is demonstrated in Figure 4. For dust with $x \gg 1$ and $|m-1| x \ll 1$, one can use the "anomalous diffraction" theory [69. For dust with $|m-1| x \ll 1$ and $|m-1| \ll 1$, one can use the Rayleigh-Gans approximation 14 to obtain the absorption and scattering cross sections [6, 39, 69]:

$$
Q_{\mathrm{abs}} \approx \frac{8}{3} \operatorname{Im}\{m x\} \quad, \quad Q_{\mathrm{sca}} \approx \frac{32|m-1|^{2} x^{4}}{27+16 x^{2}} .
$$

It is important to note that the Rayleigh-Gans approximation is invalid for modeling the X-ray scattering by interstellar dust at energies below $1 \mathrm{keV}$. This approximation systematically and substantially overestimates the intensity of the X-ray halo below $1 \mathrm{keV} 66$.

\section{Scattering of Light by Dust: Numerical Techniques}

While simple analytic expressions exist for the scattering and absorption properties of dust grains which are either very small or very large compared to the wavelength of the incident radiation (see \$5), however, in many astrophysical applications we are concerned with grains which are neither very small nor very large compared to the wavelength. Moreover, cosmic dust would in general be expected to have non-spherical, irregular shapes.

Our ability to compute scattering and absorption cross sections for nonspherical particles is extremely limited. So far, exact solutions of scattering

$\overline{13}$ At a first glance, $Q_{\text {ext }} \rightarrow 2$ appears to contradict "common sense" by implying that a large grain removes twice the energy that is incident on it! This actually can be readily understood in terms of basic optics principles: (1) on one hand, all rays impinging on the dust are either scattered or absorbed. This gives rise to a contribution of $C_{\text {geo }}$ to the extinction cross section. (2) On the other hand, all the rays in the field which do not hit the dust give rise to a diffraction pattern that is, by Babinet's principle, identical to the diffraction through a hole of area $C_{\text {geo }}$. If the detection excludes this diffracted light then an additional contribution of $C_{\text {geo }}$ is made to the total extinction cross section [6].

${ }^{14}$ The conditions for the Rayleigh-Gans approximation to be valid are $|m-1| \ll 1$ and $|m-1| x \ll 1$. The former ensures that the reflection from the surface of the dust is negligible (i.e. the impinging light enters the dust instead of being reflected); the latter ensures that the phase of the incident wave is not shifted inside the dust. For sufficiently small scattering angles, it is therefore possible for the waves scattered throughout the dust to add coherently. The intensity $(I)$ of the scattered waves is proportional to the number $(N)$ of scattering sites squared: $I \propto N^{2} \propto \rho^{2} a^{6}$ (where $\rho$ is the mass density of the dust). This is why the X-ray halos (usually within $\sim 1^{\circ}$ surrounding a distant X-ray point source; 63]) created by the small-angle scattering of X-rays by interstellar dust are often used to probe the size (particularly the large size end; 14, 67, 73]), morphology (compact or porous; 58, 66]), composition, and spatial distribution of dust. 
problems exist only for bare or layered spherical grains ("Mie theory"; 6]), infinite cylinders [52, and spheroids [3, 4, 70. The "T-matrix" (transition matrix) method, originally developed by Barber \& Yeh [5] and substantially extended by Mishchenko, Travis, \& Mackowski 59, is able to treat axisymmetric (spheroidal or finite cylindrical) grains with sizes comparable to the wavelength. The discrete dipole approximation (DDA), originally developed by Purcell \& Pennypacker [65] and greatly improved by Draine [10, is a powerful technique for irregular heterogeneous grains with sizes as large as several times the wavelength. The VIEF (volume integration of electric fields) method developed by Hage \& Greenberg [25, based on an integral representation of Maxwell's equations, is physically similar to the DDA method. The microwave analog methods originally developed by Greenberg, Pedersen \& Pedersen [21] provide an effective experimental approach to complex particles [23].

Although interstellar grains are obviously non-spherical as evidenced by the observed polarization of starlight, the assumption of spherical shapes (together with the Bruggeman or the Maxwell-Garnett effective medium theories for inhomogeneous grains; [ ) is usually sufficient in modeling the interstellar absorption, scattering and IR (continuum) emission. For IR polarization modeling, the dipole approximation for spheroidal grains is proven to be successful in many cases.

The DDA method is highly recommended for studies of inhomogeneous grains and irregular grains such as cometary, interplanetary, and protoplanetary dust particles which are expected to have a porous aggregate structure. Extensive investigations using the DDA method have been performed for the scattering, absorption, thermal IR emission, and radiation pressure properties of fluffy aggregated dust (e.g. see 22, 23, 29, 30, 31, 33, 34, 35, 36, 37, 40, 43, 56, 57, 60, 61, 71, 74, 76, 77]).

Acknowledgements I thank Drs. I. Mann and T. Mukai for their very helpful comments and support. Partial support by a Chandra Theory program and HST Theory Programs is gratefully acknowledged.

\section{References}

1. Aguirre, A.N. 1999, Intergalactic Dust and Observations of Type IA Supernovae, Astrophys. J. 512, L19

2. Andersen, A.C., Mutschke, H., Posch, T., Min, M., \& Tamanai, A. 2006, Infrared Extinction by Homogeneous Particle Aggregates of $\mathrm{SiC}, \mathrm{FeO}$ and $\mathrm{SiO}_{2}$ : Comparison of Different Theoretical Approaches, J. Quant. Spectrosc. Radiat. Transfer 100, 4

3. Asano, S., \& Sato, M. 1980, Light Scattering by Randomly Oriented Spheroidal Particles, Appl. Opt. 19, 962

4. Asano, S., \& Yamamoto, G. 1975, Light Scattering by a Spheroidal Particle, Appl. Opt. 14, 29

5. Barber, P., \& Yeh, C. 1975, Scattering of Electromagnetic Waves by Arbitrarily Shaped Dielectric Bodies, Appl. Opt. 14, 2864 
6. Bohren, C.F., \& Huffman, D. R. 1983, Absorption and Scattering of Light by Small Particles, New York: Wiley

7. Brownlee, D.E., et al. 2006, Comet 81P/Wild 2 Under a Microscope, Science, 314, 1711

8. Clayton, D.D., \& Nittler, L.R. 2004, Astrophysics with Presolar Stardust, Ann. Rev. Astro. Astrophys. 42, 39

9. Coronado, E.A., \& Schatz, G.C. 2003, Surface Plasmon Broadening for Arbitrary Shape Nanoparticles: A Geometrical Probability Approach, J. Chem. Phys. 119, 3926

10. Draine, B.T. 1988, The Discrete-Dipole Approximation and its Application to Interstellar Graphite Grains, Astrophys. J. 333, 848

11. Draine, B.T. 1990, Evolution of Interstellar Dust, in ASP Conf. Ser. 12, The Evolution of the Interstellar Medium, ed. L. Blitz (San Francisco: ASP), 193

12. Draine, B.T. 2003, Scattering by Interstellar Dust Grains. I. Optical and Ultraviolet, Astrophys. J. 598, 1017

13. Draine, B.T., \& Lee, H.M. 1984, Optical Properties of Interstellar Graphite and Silicate Grains, Astrophys. J. 285, 89

14. Draine, B.T., \& Tan, J.C. 2003, The Scattered X-Ray Halo around Nova Cygni 1992: Testing a Model for Interstellar Dust, Astrophys. J. 594, 347

15. Dwek, E. 2004a, Galactic Center Extinction: Evidence of Metallic Needles in the General Interstellar Medium, Astrophys. J. 611, L109

16. Dwek, E. 2004b, The Detection of Cold Dust in Cassiopeia A: Evidence for the Formation of Metallic Needles in the Ejecta, Astrophys. J. 607, 848

17. Fabian, D., Henning, Th., Jäger, C., Mutschke, H., Dorschner, J., \& Wehrhan, O. 2001, Steps toward Interstellar Silicate Mineralogy. VI. Dependence of Crystalline Olivine IR Spectra on Iron Content and Particle Shape, Astron. Astrophys. 378, 228

18. Farafonov, V.G. 2001, Light Scattering by Multilayer Nonconfocal Ellipsoids in the Rayleigh Approximation, Opt. Spectrosc. 90, 574

19. Gilra, D.P. 1972, Collective Excitations and Dust Particles in Space, in Scientific Results from the Orbiting Astronomical Observatory (OAO-2), 310, 295

20. Greenberg, J.M. 1982, What Are Comets Made of? in Comets, ed. L.L. Wilkening (Tuscon: Univ. of Arizona Press), 131

21. Greenberg, J.M., Pedersen, N.E., \& Pedersen, J.C. 1961, Microwave Analog to the Scattering of Light by Nonspherical Particles, J. Appl. Phys. 32, 233

22. Grün, E., et al. 2005, Kuiper prize lecture: Dust Astronomy, Icarus, 174, 1

23. Gustafson, B.A.S., \& Kolokolova, L. 1999, A Systematic Study of Light Scattering by Aggregate Particles Using the Microwave Analog Technique: Angular and Wavelength Dependence of Intensity and Polarization, J. Geophys. Res. 104, 31711

24. Habbal, S.R., et al. 2003 On the Detection of the Signature of Silicon Nanoparticle Dust Grains in Coronal Holes, Astrophys. J. 592, L87

25. Hage, J.I., \& Greenberg, J.M. 1990, A Model for the Optical Properties of Porous Grains, Astrophys. J. 361, 251

26. Henyey, L.G., \& Greenstein, J.L. 1941, Diffuse Radiation in the Galaxy, Astrophys. J. 93, 70

27. Hoyle, F., \& Wickramasinghe, N.C. 1988, Metallic Particles in Astronomy, Astrophys. Space Sci., 147, 245

28. Jackson, J.D. 1998, Classical Electrodynamics (3rd ed), New York: Wiley 
29. Kimura, H., Okamoto, H., \& Mukai, T. 2002, Radiation Pressure and the Poynting-Robertson Effect for Fluffy Dust Particles, Icarus 157, 349

30. Kimura, H., Kolokolova, L., \& Mann, I. 2003, Optical Properties of Cometary Dust: Constraints from Numerical Studies on Light Scattering by Aggregate Particles, Astron. Astrophys. 407, L5

31. Kimura, H., Kolokolova, L., \& Mann, I. 2006, Light Scattering by Cometary Dust: Numerically Simulated with Aggregate Particles Consisting of Identical Spheres, Astron. Astrophys. 449, 1243

32. Kissel, J., et al. 2007, COSIMA - High Resolution Time-of-Flight Secondary Ion Mass Spectrometer for the Analysis of Cometary Dust Particles onboard Rosetta, Space Sci. Rev. 128, 823

33. Köhler, M., Kimura, H., \& Mann, I. 2006, Applicability of the Discrete-Dipole Approximation to Light-Scattering Simulations of Large Cosmic Dust Aggregates, Astron. Astrophys. 448, 395

34. Köhler, M., Minato, T., Kimura, H., \& Mann, I. 2007, Radiation Pressure Force Acting on Cometary Aggregates, Adv. Space Res. 40, 266

35. Kolokolova, L., Hanner, M.S., Levasseur-Regourd, A.-C., \& Gustafson, B.A.S. 2004, Physical Properties of Cometary Dust from Light Scattering and Thermal Emission, Comets II, 577

36. Kozasa, T., Blum, J., \& Mukai, T. 1992, Optical Properties of Dust Aggregates. I. Wavelength Dependence, Astron. Astrophys. 263, 423

37. Kozasa, T., Blum, J., Okamoto, H., \& Mukai, T. 1993, Optical Properties of Dust Aggregates. II. Angular Dependence of Scattered Light, Astron. Astrophys. 276, 278

38. Landau, L.D., Lifshitz, E.M., \& Pitaevskii, L.P. 2000, Electrodynamics of Continuous Media (2nd ed.), Oxford: Pergamon

39. Laor, A., \& Draine, B.T. 1993, Spectroscopic Constraints on the Properties of Dust in Active Galactic Nuclei, Astrophys. J. 402, 441

40. Lasue, J., \& Levasseur-Regourd, A.C. 2006, Porous Irregular Aggregates of SubMicron Sized Grains to Reproduce Cometary Dust Light Scattering Observations, J. Quant. Spectrosc. Radiat. Transfer 100, 220

41. Ledoux, G., et al. 1998, Silicon as a Candidate Carrier for ERE, Astron. Astrophys. 333, L39

42. Lee, H.M., \& Draine, B.T. 1985, Infrared Extinction and Polarization due to Partially Aligned Spheroidal Grains - Models for the Dust toward the BN Object, Astrophys. J. 290, 211

43. Levasseur-Regourd, A.C., Mukai, T., Lasue, J., \& Okada, Y. 2007, Physical Properties of Cometary and Interplanetary Dust, Planet. Space Sci. 55, 1010

44. Li, A. 2003a, On TiC Nanoparticles as the Origin of the $21 \mu \mathrm{m}$ Emission Feature in Post-Asymptotic Giant Branch Stars, Astrophys. J. 599, L45

45. Li, A. 2003b, Cosmic Needles versus Cosmic Microwave Background Radiation, Astrophys. J. 584, 593

46. Li, A. 2005a, Can Fluffy Dust Alleviate the Subsolar Interstellar Abundance Problem?, Astrophys. J. 622, 965

47. Li, A. 2005b, On the Absorption and Emission Properties of Interstellar Grains, in "The Spectral Energy Distribution of Gas-Rich Galaxies: Confronting Models with Data", ed. C.C. Popescu, \& R.J. Tuffs, AIP Conf. Ser. 761, 163

48. Li, A., \& Draine, B.T. 2002, Are Silicon Nanoparticles an Interstellar Dust Component? Astrophys. J. 564, 803 
49. Li, A., Greenberg, J.M., \& Zhao, G. 2002, Modelling the Astronomical Silicate Features - I. On the Spectrum Subtraction Method, Mon. Not. Roy. Astron. Soc. 334,840

50. Li, A., Misselt, K.A., \& Wang, Y.J. 2006, On the Unusual Depletions toward Sk 155 or What Are the Small Magellanic Cloud Dust Grains Made of?, Astrophys. J. 640, L151

51. Li, A., Ortega, J., \& Lunine, J.I. 2008, BD+20 30\%: Attogram Dust or Extreme Asteroidal Collisions?, in preparation

52. Lind, A.C., \& Greenberg, J.M. 1966, Electromagnetic Scattering by Obliquely Oriented Cylinders, J. Appl. Phys. 37, 3195

53. Mann, I. 2007a, Evolution of Dust and Small Bodies: Physical Processes, in Small Bodies in Planetary Systems, ed. I. Mann, A.M. Nakamura, \& T. Mukai, (Springer: Berlin), 180

54. Mann, I. 2007b, Nanoparticles in the Inner Solar System, Planet. Space Sci. 55, 1000

55. Mann, I., \& Murad, E. 2005, On the Existence of Silicon Nanodust near the Sun, Astrophys. J. 624, L125

56. Mann, I., Okamoto, H., Mukai, T., Kimura, H., \& Kitada, Y. 1994, Fractal Aggregate Analogues for Near Solar Dust Properties, Astron. Astrophys. 291, 1011

57. Mann, I., Kimura, H., \& Kolokolova, L. 2004, A Comprehensive Model to Describe Light Scattering Properties of Cometary Dust, J. Quant. Spectrosc. Radiat. Transfer 89, 291

58. Mathis, J.S., Cohen, D., Finley, J.P., \& Krautter, J. 1995, The X-Ray Halo of Nova V1974 Cygni and the Nature of Interstellar Dust, Astrophys. J. 449, 320

59. Mishchenko, M.I., Travis, L.D., \& Macke, A. 1996, Scattering of Light by Polydisperse, Randomly Oriented, Finite Circular Cylinders, Appl. Opt. 35, 4927

60. Mukai, T., Ishimoto, H., Kozasa, T., Blum, J., \& Greenberg, J.M. 1992, Radiation Pressure Forces of Fluffy Porous Grains, Astron. Astrophys. 262, 315

61. Okada, Y., Nakamura, A.M., \& Mukai, T. 2006, Light Scattering by Particulate Media of Irregularly Shaped Particles: Laboratory Measurements and Numerical Simulations, J. Quant. Spectrosc. Radiat. Transfer 100, 295

62. Ossenkopf, V., Henning, Th., \& Mathis, J.S. 1992, Constraints on Cosmic Silicates, Astron. Astrophys. 261, 567

63. Overbeck, J.W. 1965, Small-Angle Scattering of Celestial X-Rays by Interstellar Grains, Astrophys. J. 141, 864

64. Purcell, E.M. 1969, On the Absorption and Emission of Light by Interstellar Grains, Astrophys. J. 158, 433

65. Purcell, E.M., \& Pennypacker, C.R. 1973, Scattering and Absorption of Light by Nonspherical Dielectric Grains, Astrophys. J. 186, 705

66. Smith, R.K., \& Dwek, E. 1998, Soft X-Ray Scattering and Halos from Dust, Astrophys. J. 503, 831

67. Smith, R.K., Edgar, R.J., \& Shafer, R.A. 2002, The X-Ray Halo of GX 13+1, Astrophys. J. 581, 562

68. Song, I., Zuckerman, B., Weinberger, A.J., \& Becklin, E.E. 2005, Extreme Collisions between Planetesimals as the Origin of Warm Dust around a Sun-like Star, Nature 436, 363

69. van de Hulst, H.C. 1957, Light Scattering by Small Particles, New York: John Wiley \& Sons 
70. Voshchinnikov, N.V., \& Farafonov, V.G. 1993, Optical Properties of Spheroidal Particles, Astrophys. Space Sci. 204, 19

71. Wilck, M., \& Mann, I. 1996, Radiation Pressure Forces on "Typical" Interplanetary Dust Grains, Planet. Space Sci. 44, 493

72. Witt, A.N., Gordon, K.D., \& Furton, D.G. 1998, Silicon Nanoparticles: Source of Extended Red Emission? Astrophys. J. 501, L111

73. Witt, A.N., Smith, R.K., \& Dwek, E. 2001, X-Ray Halos and Large Grains in the Diffuse Interstellar Medium, Astrophys. J. 550, L201

74. Wolff, M.J., Clayton, G.C., \& Gibson, S.J. 1998, Modeling Composite and Fluffy Grains. II. Porosity and Phase Functions, Astrophys. J. 503, 815

75. Wright, E.L. 1982, Thermalization of Starlight by Elongated Grains - Could the Microwave Background Have Been Produced by Stars? Astrophys. J. 255, 401

76. Xing, Z.F., \& Hanner, M.S. 1999, Light Scattering by Aggregate Particles, Astron. Astrophys. 324, 805

77. Yanamandra-Fisher, P.A., \& Hanner, M.S. 1999, Optical Properties of Nonspherical Particles of Size Comparable to the Wavelength of Light: Application to Comet Dust, Icarus 138, 107 